\title{
TRAYECTORIA ACADÉMICA DEL PROFESOR ALFREDO MORALES GIL
}

\section{LA FORMACIÓN COMO GEÓGRAFO EN LA UNIVERSIDAD DE MURCIA}

Alfredo Morales Gil es un claro ejemplo de decidida vocación y vivencia de la Geografía. Su primer contacto académico con esta disciplina tiene lugar cuando cursa los estudios de Magisterio en la entonces denominada Escuela Normal de Murcia, en la que es alumno del profesor Isidoro Reverté Salinas, un gran docente que practicaba la enseñanza de la geografía en el campo y que contribuye a estimular la pasión por el conocimiento de su entorno regional. Una vocación que alimenta con la lectura de los relatos científicos sobre las exploraciones de África y de las regiones polares. Se convierte así Alfredo Morales en un apasionado viajero virtual, a través de esas lecturas y, tras una etapa inicial en el desempeño de su puesto como profesor de enseñanza primaria, se matricula en la Universidad de Murcia, donde cursa la especialidad de Geografía e Historia, en la Facultad de Filosofía y Letras, en la que sería alumno del Dr. Vilá Valentí, uno de los referentes de la Geografía española de la época. Allí conoce a los entonces jóvenes profesores ayudantes Horacio Capel y Francisco Calvo García-Tornel, con los que comparte su etapa de formación académica. De hecho, fueron las enseñanzas de Horacio Capel, en jornadas de campo, las que le deciden a acometer su primer trabajo de investigación, referido a los nuevos regadíos de la cuenca del río Segura y la ampliación de las superficies y del aprovechamiento agrícola basado en el regadío, desde la huerta tradicional hacia áreas contiguas, a raíz del Decreto de 1953 y la consiguiente ordenación de los aprovechamientos hidráulicos de la cuenca de este río.

En esa misma etapa de formación del profesor Morales Gil, como profesor contratado, llega al Departamento de Geografía de la Universidad de Murcia el Dr. Rosselló Verger, el cual sería esencial en su orientación geográfica, sobre todo por la impronta que Rosselló le aporta sobre el papel de la Geografía Física, para alcanzar la visión integral y de síntesis de la Geografía que siempre ha sido una de las claves del modo en que el profesor Morales ha entendido e interpretado esta disciplina. Dos conceptos esenciales estarán presentes en toda su 
producción geográfica. Uno es el paisaje, entendido como la forma del espacio geográfico que reproduce la síntesis de los procesos físicos y humanos desarrollados a través del tiempo, y su papel en la identidad del territorio. El otro es la región, como categoría analítica que le permite sentar las bases para el análisis geográfico. La idea de entender e interpretar el paisaje geográfico le conduce en esa etapa a asistir a tres ediciones anuales de los Cursos de Trabajo de Campo sobre Geomorfología Estructural que organizaba el Dr. García Fernández en la comarca burgalesa de Las Loras y que se convirtieron, durante años, en un excelente medio para el aprendizaje de la Geografía Física, y del conocimiento geográfico en general, a través de la práctica y del contacto directo con el medio.

La etapa murciana del profesor Morales coincide también con la configuración en esa Universidad de un grupo de geógrafos que iniciaban sus investigaciones y que definieron líneas esenciales para el conocimiento de la realidad geográfica regional, desde diferentes dimensiones de análisis. A los profesores Calvo y Capel, aunque éste se trasladaría a la Universidad de Barcelona, se añaden otros investigadores como López Bermúdez, López Ontiveros y Juan Moreno, que iniciaban sus tesis doctorales y creaban la idea de grupo de investigación, en un contexto bastante más difícil que el actual para acometer proyectos de investigación en la universidad española. Ese grupo llegaría a realizar aportaciones esenciales para el conocimiento y análisis espacial de su entorno, principalmente con sus respectivas tesis, pero también de forma conjunta, como fue la contribución a la división comarcal de la entonces provincia de Murcia, desde los planteamientos de la Geografía. De esos años, destaca también el esfuerzo de este grupo de investigadores por conocer la producción científica de la Geografía que se hacía fuera de España y que se materializó en el acceso a obras de referencia esencial que marcaron la pauta en aquel momento, como la Geografía de la Pléyade, editada en Francia en 1966. Además de la influencia que reciben de algunos geógrafos franceses y alemanes, como F. Geiger, A. Humbert y R. Courtot, que visitaban el Departamento de Geografía de la Universidad de Murcia con motivo de sus investigaciones referidas al sureste ibérico.

En 1972, defiende Alfredo Morales su tesis sobre el Altiplano de JumillaYecla, una de las primera tesis doctorales del Departamento de Geografía de la Universidad de Murcia y la primera dirigida por el Dr. Rosselló Verger, en el marco del paradigma regional al uso en aquel momento, aportando una visión integral de los procesos físicos y humanos que configuran un espacio geográfico que este trabajo delimitó, con una importante aplicación de técnicas cartográficas que contribuirán a que la labor realizada haya perdurado en el tiempo, como fundamento de su diferenciación territorial.

De esta etapa de formación en la Universidad de Murcia surgen sus primeras aportaciones a congresos así como publicaciones, especialmente las que 
realiza en la revista Papeles del Departamento de Geografía, de cuya secretaría de redacción se ocupó el Profesor Morales durante un tiempo.

\section{LA UNIVERSIDAD DE LA LAGUNA Y EL CONOCIMIENTO GEOGRÁFICO DEL MEDIO INSULAR CANARIO}

Tras acceder a la condición de profesor adjunto, en 1975, Alfredo Morales toma posesión de su plaza en la Universidad de La Laguna, donde se encuentra con una realidad geográfica muy diferente a la de su tierra de origen. Desde el primer momento, su dedicación de geógrafo se enfrenta al reto de entender y analizar la configuración del paisaje, fundamentado esencialmente en el origen volcánico de las islas y en sus condiciones climáticas. Con esta finalidad, simultanea la labor docente en las aulas con el trabajo de campo, junto con los profesores Martín Galant, Quirantes y Rodríguez Brito. En la vertiente académica, se encargaría de la coordinación del plan de estudios de Geografía e Historia, en el marco de un contexto general en España en el que se estaba produciendo la institucionalización y especificidad de la Geografía, como especialidad, a partir de los antiguos planes genéricos en Filosofía y Letras. Mientras que su dedicación al trabajo de campo, además de avanzar en su conocimiento geográfico del medio insular canario, le aporta resultados apasionantes, sobre todo por haber encontrado huellas del modelado periglaciar en las Cañadas del Teide, en Tenerife. Algo que sorprendió a algún maestro de la época, como el propio Dr. García Fernández. Resultado de estas investigaciones es un libro monográfico dedicado al tema y, por su trascendencia en la Geografía, la aportación realizada, junto con Quirantes y Martín, al Colloque sur le preglaciare d'altitud du Mediterranéen, celebrado en Estrasburgo, en septiembre de 1977, organizado por el Dr. J. Tricart, uno de los mayores referentes internacionales de la Geografía Física de la época. Una línea de trabajo que desarrollaría el profesor Martínez de Pisón durante su etapa como Catedrático de Geografía Física de la Universidad de La Laguna, a partir del curso 1977-78.

En síntesis, los años de estancia en Canarias suponen para el profesor Morales una fuente de conocimientos geográficos que contribuyen a su formación académica, a partir de la comprensión de un medio diferente. Pero también significan, en el plano personal, la apertura a nuevos horizontes vitales en el desempeño de la condición de geógrafo que siempre la ha acompañado.

\section{UN REFERENTE DE LA GEOGRAFÍA ESPAÑOLA DESDE SU LABOR EN LA} UNIVERSIDAD DE AlicANTE

Alfredo Morales se incorpora al campus alicantino en octubre de 1977 -en ese momento todavía dependiente de la Universidad de Valencia- donde de inmediato participa en las tareas que, con gran acierto, llevaba adelante el Dr. Gil 
Olcina, como Decano de la Facultad de Filosofía y Letras, en aras de la creación de la especialidad de Geografía. De hecho, uno de los primeros retos para el profesor Morales fue acometer la reforma del plan de estudios para dar cabida a la sección de Geografía e Historia y a la especialidad de Geografía, como ya había hecho en La Laguna. De igual modo, la consolidación de la Geografía requería la creación de una unidad departamental específica, labor que lleva a cabo Alfredo Morales, con la participación de un núcleo de profesorado con el que contaba en aquel momento, reducido pero comprometido en el mismo empeño, con compañeros como Antonio Ramos, José Costa, Enrique Matarredona y, poco después, Cipriano Juárez; siempre con el apoyo del profesor Gil Olcina, que pronto pasaría a ser Presidente de la Comisión Gestora de la Universidad de Alicante y que proyectaba su obra como uno de los grandes maestros de la geografía española.

De manera paralela a la actividad docente y a la puesta en marcha del Departamento de Geografía, entre 1980 y 1985, Alfredo Morales desempeña el cargo de Vicerrector de Extensión Universitaria de la recién creada Universidad de Alicante, como parte del equipo de gobierno del Rector Gil Olcina, con unas competencias que abarcaban las infraestructuras y la planificación del campus lucentino, entre cuyas tareas esenciales estaba la remodelación de los antiguos edificios del campamento militar preexistente al campus y la construcción de otros nuevos en los terrenos cedidos para la ampliación del campus de Rabasa, donde se irían albergando nuevas Facultades que se iban creando, en la fase de despegue de la UA. Una tarea que marcaría en el futuro la planificación del emplazamiento universitario y en la que siempre destacó el profesor Morales por su sentido práctico y capacidad resolutiva de problemas en lo que fue el proceso de puesta en marcha del campus alicantino. Tarea que mereció, por Resolución Rectoral, el reconocimiento con la Medalla de Oro de la Universidad, en 1984.

En el ámbito de su quehacer como geógrafo, la década de los ochenta del siglo XX supone para el profesor Morales Gil, ya consolidado como Catedrático de Universidad, una etapa de nuevos retos y proyectos esenciales para el futuro de la Geografía en Alicante. Destaca la creación de un aula especializada de Cartografía, con un bagaje relevante de fondos, tanto cartográficos como fotogramétricos, poco usuales en aquel momento en los departamentos de Geografía españoles. Entre esos fondos, merece una mención especial la disponibilidad del vuelo americano de 1956, un material clave para analizar procesos de transformación del territorio, que resultó decisivo para algunas de las investigaciones que pronto se pusieron en marcha, en temas diversos, como los cambios en los paisajes agrarios, configuración de áreas turísticas, estudios del relieve y áreas naturales, entre otros aspectos que motivaron el interés por encauzar su investigación entre jóvenes licenciados/as que iban saliendo de las aulas y que se incorporaban al Departamento como becarios de investigación, en bastantes 
casos bajo la dirección académica del Dr. Morales Gil (Box Amorós, Brú Ronda, Vera Rebollo, Ponce Herrero, Marco Molina, entre otros).

La eclosión de esta generación de investigadores contó además con el privilegio, en aquel momento, de disponer de una biblioteca especializada en Geografía, con fondos que el Dr. Morales se preocupó de conseguir, generando un verdadero reservorio de bibliografía especializada, con los títulos más relevantes (monografías y revistas de investigación) que iban surgiendo en la producción geográfica en el ámbito nacional e internacional. La tarea orientada a configurar un verdadero núcleo de investigación en Geografía se ve reforzada con la creación del Instituto Universitario de Geografía y la revista Investigaciones Geográficas, que pronto se convertiría en referencia para geógrafos nacionales e internacionales y que permitía difundir los resultados de trabajos realizados en los departamentos de Análisis Geográfico Regional y Geografia Humana de la Universidad de Alicante.

La actividad investigadora impulsada por el profesor Morales Gil en esta etapa se vio fortalecida por la presencia estacional, en esta Universidad, del Dr. García Fernández, prestigioso Catedrático de Geografía Física de la Universidad de Valladolid, una de las personalidades más influyentes en la Geografía española de la época, que tuvo su impronta en trabajos realizados por miembros del Instituto Universitario de Geografía. En el mismo sentido de robustecimiento del núcleo investigador, el profesor Morales participó en convocatorias públicas para la consecución de proyectos de investigación competitivos realizados en equipo, en líneas referidas a usos del agua, organización del espacio y riesgo de inundaciones, entre otras. Mientras que, paralelamente y con la dirección conjunta del Dr. Gil Olcina, se acometieron contratos con organismos públicos (Diputación Provincial, Ayuntamientos) referidos al estudio de aspectos relacionados con la ordenación del territorio y riesgos, en el ámbito de la Geografía Aplicada, que abrieron una nueva dimensión de trabajo para los geógrafos y, sobre todo, nuevos horizontes para la práctica profesional de la Geografía, en una época en la que apenas se hablaba de esta vertiente.

Las líneas prioritarias de investigación del Instituto de Geografía, dirigido por Gil Olcina, y del Departamento conducido por Alfredo Morales, generaron una importante transferencia de resultados, mediante la celebración de diversos coloquios, a lo largo de más de dos décadas, que marcaron la pauta en España en el conocimiento científico de temas relevantes para la Geografía. Desde el Coloquio sobre La propiedad de la tierra en España (1980), se organizaron los de Demanda y Economía del Agua (1986), Avenidas fluviales e inundaciones en la cuenca del Mediterráneo (1988), Hitos históricos de los regadíos españoles (1990), Medio siglo de cambios agrarios en España (1991), Planificación Hidráulica en España (1994), Usos del Agua (1998), Causas y consecuencia de las sequías (2000), Insuficiencias hídricas y plan hidrológico nacional (2001), 
Riesgo de inundaciones en el mediterráneo occidental (2004), entre otros, todos publicados en formato de libro, con participación de expertos nacionales e internacionales en las materias.

El profesor Morales participó, como ponente, en decenas de coloquios nacionales e internacionales, sobre temas de su especialidad, entre los que destacan los organizados por la Unión Geográfica Internacional, la Asociación de Geógrafos Españoles, el Centre Européen de Coordination de Recherches et de Documentation en Sciences Sociales, de Viena; el CNRS de Francia; o la Casa de Velázquez, entre otros muchos.

En el plano de la docencia, Alfredo Morales Gil ha mantenido, a lo largo de toda su trayectoria, un firme compromiso en la defensa de la Geografía y de su papel formativo. Destacó por su labor en el aula, donde su modo de entender y enseñar la Geografía le llevó a despertar la vocación por el análisis geográfico entre el alumnado y a ser reconocido como un gran docente. Pero, además, contribuyó a la defensa de la disciplina y a su presencia en los planes de estudio de los que fue coordinador en distintas universidades y diferentes contextos. En una primera etapa, tanto en La Laguna como en Alicante, tuvo la responsabilidad de sacar adelante nuevos planes de estudio que supusieron la incorporación plena de la especialidad de Geografía, dentro de la sección de Geografía e Historia, con la consiguiente integración de las materias clave para la formación del alumnado, siempre desde una visión de la Geografía basada en la interacción de procesos físicos y humanos, lo que le llevó a defender la presencia de todas las asignaturas que contribuyeran a esa formación integral, de síntesis, propia de la Geografía, y a dar una significativa importancia a las técnicas cartográficas, como herramienta clave en la formación del geógrafo. Buscó además la interrelación entre la vertiente docente y la faceta investigadora, desde la idea de que una formación de calidad, sobre la base de unos sólidos conocimientos y manejo de métodos, es determinante para hacer investigación. A la vez que la investigación permite alcanzar conocimientos científicos y avanzar en el modo de formular y estudiar los problemas. Sobre este enfoque, su objeto esencial de estudio fue el paisaje geográfico y el análisis de las dinámicas y procesos que han llevado a su configuración, así como el modo en que se producen las transformaciones territoriales, en distintos contextos sociales y económicos, sobre ámbitos espaciales concretos. Desde esa preocupación centrada en el análisis espacial, Morales Gil primó siempre la faceta del trabajo de campo y el conocimiento directo del medio, uno de sus grandes puntos fuerte como formador ya que, guiado por una sorprendente intuición para comprender realidades del entorno territorial, era capaz de interpretar los hechos geográficos sobre el terreno con una maestría que motivaba e inspiraba a los que tuvimos la suerte de aprender con él. 
Con independencia del objeto específico de los trabajos de investigación que han centrado su interés y del enfoque paradigmático con el que ha estudiado la realidad geográfica, prevalece su visión de síntesis de la Geografía, otorgando importancia a los dos grandes conjuntos que siempre han definido el conocimiento en esta disciplina, la Geografía Física y la Humana, y a la región como ámbito y categoría de análisis para su interpretación. De ahí su idea, manifestada en las últimas revisiones de planes de estudio, por incluir una troncalidad común para los estudios de Geografía, lo que Morales Gil considera unos pilares básicos en la formación que atribuyen bases sólidas de conocimiento científico sobre las cuales se ha de reconocer la proyección social de la Geografía.

No podría cerrarse su etapa creativa en la Geografía sin aludir a su interés por fomentar la relación e interacción con otros grupos de investigación para poder así entender el análisis de distintas realidades geográficas y los procesos de organización territorial, en diferentes contextos, a la vez que validar y contrastar métodos y técnicas de análisis espacial. Resultado de su faceta de relaciones externas, en el ámbito nacional e internacional, son sus publicaciones con colegas de otras universidades, del mismo modo que lo fue su participación en la acción integrada que el Instituto de Geografía de la UA llevó a cabo con el Departamento de Geografía «Giuseppe Morandini» de la Universidad de Padua, a raíz de la cual realizó estancias de investigación y seminarios conjuntos con profesores de esa Universidad, sobre la base de líneas de investigación de interés compartido, especialmente la relativa a usos y gestión del agua, en las que el profesor Morales participó activamente junto a especialistas de otras universidades europeas como las de Saint-Etienne, Marsella; y el SOAS de la Universidad de Londres.

En apretado balance final, la trayectoria de Alfredo Morales Gil es representativa de la generación de grandes maestros de nuestra disciplina, destacable por su forma de transmitir las enseñanzas y de entender la Geografía. De su faceta como investigador, destaca por sus aportaciones referidas al análisis de los factores que explican cambios paisajísticos y transformaciones territoriales, así como a la interpretación y estudio de las dinámicas espaciales, siempre desde una visión de síntesis de los hechos físicos y humanos que configuran procesos y modelos de organización espacial. De su impronta y magisterio da buena cuenta el hecho de haber creado un grupo de profesores e investigadores que desempeñan sus funciones docente e investigadora en el seno de la comunidad académica. Su trayectoria define a un profesor universitario cuya talla académica convive con unos valores humanos que motivan el respeto y el afecto de la comunidad geográfica española. Y prueba de ello es el testimonio elocuente hacia su obra y su legado que se recoge en las numerosas muestras de reconocimiento recibidas, por parte de discípulos y compañeros de profesión de diferentes universidades, en el presente libro homenaje. 


\section{LÍNEAS DE TRABAJO PRINCIPALES EN LA TRAYECTORIA INVESTIGADORA DE} Alfredo Morales GiL

Un currículo científico es la expresión de una serie de temáticas de trabajo por las que se apuesta a lo largo de la vida académica. Se trata de querencias temáticas que confluyen en la trayectoria personal del universitario, casi siempre por elección propia en la que juega el conocimiento previo del tema por circunstancias personales o por pertenencia al ámbito territorial objeto de estudio; pero a veces, algunos temas llegan por el propio azar, por el desarrollo de hechos que nos sitúa ante cuestiones sobre las que entendemos que merece la pena indagar dentro de un campo científico. En el caso del profesor Alfredo Morales confluyen estas dos razones en la justificación de un conjunto de líneas de trabajo que han marcado su trayectoria académica y que, en esencia, se pueden resumir en las siguientes: la ordenación de los paisajes del sureste peninsular y los efectos de su desorganización; la actividad agraria y, con especial atención, las nuevas formas de agricultura intensiva y sus consecuencias socioeconómicas y territoriales; los recursos y los usos del agua y su relación con el desarrollo de actividades económicas (agraria, urbano-turística) en la fachada mediterránea peninsular; las infraestructuras de comunicación como ejes vertebradores del desarrollo regional; los riesgos naturales de fuerte impronta territorial y efectos económicos, como inundaciones y sequías; el medio físico como armazón básico de la labor del ser humano; la geografía regional como manera de comprender las dinámicas territoriales; y el valor de los aspectos históricos del territorio como elementos que subyacen al desarrollo de procesos ambientales, sociales y económicos contemporáneos.

En todas ellas, la labor investigadora del profesor Alfredo Morales se ha desarrollado bajo una serie de principios geográficos, de los que ha hecho gala en su vida universitaria: la necesaria ordenación racional del territorio, donde se integren los elementos del medio físico y la acción del ser humano de forma complementaria; la importancia de los recursos naturales como elemento esencial para el desarrollo de las sociedades en los territorios; el papel de las infraestructuras como ejes vertebradores del funcionamiento de las regiones; el obligado respeto al medio físico como soporte de las actuaciones humanas; la importancia de los paisajes como manifestación de la acción del ser humano en un medio y en un momento histórico. Y junto a estos principios, el valor del trabajo de campo, de la observación directa de los hechos, como herramienta fundamental para comprender fenómenos y procesos que ocurren en el espacio geográfico. Estas han sido las sólidas bases conceptuales sobre las que se ha asentado una labor académica de varias décadas que deja en herencia la labor de una serie de discípulos que han querido seguir unos modos y maneras de hacer 
geografía arraigada en la tradición humanista pero que siempre ha cultivado el papel del geógrafo como profesional de la organización de los territorios.

Una de las primeras publicaciones científicas del profesor Alfredo Morales se relacionan con una de las temáticas que cultivará durante toda su carrera académica: la Geografía Regional y las divisiones del espacio geográfico. Se trata de un artículo sobre la división comarcal de la entonces provincia de Murcia, editado en Papeles de Geografia de la Universidad de Murcia en 1969. La propia realización de la tesis doctoral sobre el Altiplano de Jumilla-Yecla, defendida en 1972, en el contexto de la época de elaboración de trabajos doctorales sobre ámbitos regionales o comarcales de nuestro país, constituye un hito principal en su actividad investigadora. Este trabajo será objeto de posteriores ediciones, bajo el formato de capítulo de libro, en obras colectivas sobre la Región de Murcia. Por su parte, sus conocimientos de la realidad geográfica murciana serán la base de la preparación de una serie de trabajos sobre geografía regional de Murcia, editados como monografías o como parte de obras colectivas. Una línea de trabajo cultivada con especial dedicación e interés por el profesor Morales ha correspondido a la preparación y coordinación de Atlas temáticos sobre espacios regionales (Murcia, Comunidad Valenciana), puesto que la cartografía, en su modo de entender la labor geográfica, es el verdadero lenguaje del geógrafo y elemento esencial para el conocimiento de los rasgos básicos de los espacios regionales de la fachada este y, específicamente, del sureste de la península Ibérica. Igualmente, desde fechas tempranas de su trayectoria académica, dedicará algunos trabajos a valorar el papel de la excursión como método docente para el conocimiento de los espacios regionales. No en vano el profesor Morales, como responsable de la asignatura Geografía de España a lo largo de sus años de docencia en la Universidad de Alicante, será el encargado de la organización de los viajes anuales de «geografía regional» que se llevarían a cabo con los alumnos de especialidad de la titulación, por las diversas regiones de nuestro país, desde una concepción de la docencia geográfica heredera de la tradición «institucionista», que tanto ha contribuido a la formación de generaciones de geógrafos que han pasado por sus aulas.

En 1969 aparecerá otro trabajo que marcará la trayectoria investigadora posterior del profesor Alfredo Morales. Es su estudio sobre el riego con aguas de avenida, editado en Papeles de Geografia y que abrirá dos nuevas líneas de investigación en el propio trabajo del profesor Morales: los paisajes agrarios de áreas semiáridas y áridas del sureste ibérico; y la peligrosidad climática y las formas de adaptación tradicionales a la misma característica, igualmente, de este espacio geográfico, y en particular el análisis de sus dos extremos atmosféricos más significativos: lluvias torrenciales y secuencias de sequía.

La primera línea de investigación, la ordenación de los paisajes del sureste peninsular, su evolución y los efectos (paisajísticos, patrimoniales, ambientales 
y territoriales) de su desorganización ha sido una constante a lo largo de toda su trayectoria investigadora y en ella queda bien patente la visión integradora del profesor Morales. En los trabajos que integran esta temática se encuentra el necesario análisis de la interacción entre los factores físicos y humanos que dan lugar a una particular sistematización de las laderas y los barrancos que aprovechan la escorrentía superficial y los caudales circulantes por ramblas y barrancos, con ocasión de precipitaciones de intensidad horaria. Se indaga en el origen y la regulación de este aprovechamiento desde época romana y se profundiza en la evolución que registra y que culmina en una progresiva desarticulación, a consecuencia de los cambios económicos y territoriales desarrollados a partir de mediados de los años cincuenta y el incremento de la disponibilidad de recursos hídricos de aportación regular. El profesor Morales estudia, asimismo, la acentuación de una serie de procesos físicos (pérdida de suelos o de la capacidad de laminación de las ondas de crecidas, entre otros) debido a la desaparición de los beneficios ambientales asociados a esta sistematización. Sus trabajos en esta línea de investigación, han contribuido al conocimiento de un modo ordenación del espacio agrario, desconocido en buena parte del territorio nacional debido a las singulares condiciones climáticas del ámbito en que se desarrolla (sureste ibérico), donde el ser humano ha tenido que adaptarse a una natural escasez de recursos hídricos. En términos actuales, estamos ante lo que se denominaría un desarrollo sostenible, acorde con los rasgos del medio físico donde tiene lugar, cuyo atinado análisis, en un buen número de trabajos llevados a cabo por el profesor Morales, ha despertado el interés de investigadores de diferentes disciplinas (ingenieros agrónomos, sociólogos, ambientólogos, entre otros) españoles y extranjeros. Esta visión del medio y de la adaptación al mismo por parte del ser humano entronca, asimismo, con la defensa del significado y relevancia de estos paisajes desde una perspectiva territorial, patrimonial y ambiental, plasmada en algunas de sus publicaciones más recientes, donde se destaca la necesidad de trasmitir a la sociedad el valor cultural de unos paisajes singulares y únicos.

En la línea de investigación dedicada al estudio de extremos atmosféricos y sus efectos socioeconómicos y territoriales destacan numerosos trabajos (artículos, libros, capítulos de libro) y proyectos y contratos de investigación en los que participa el profesor Morales, coordinando y orientando la labor de los equipos de trabajo. Un hito principal en esta línea de investigación sobre eventos extremos, sus causas y consecuencias, tiene lugar con el desarrollo de la «gota fría» de octubre de 1982, que deja un rastro de desolación y víctimas en la propia ciudad de Alicante. El recorrido de campo en primera persona con la cámara fotográfica siempre dispuesta y la libreta de anotación de los hechos que se van observando en los espacios afectados para buscar interpretaciones posteriores sobre lo ocurrido, marcará un modo de hacer en la investigación sobre riesgos climáticos en el entonces Departamento de Geografía de la Universidad de 
Alicante. Sobresalen en esta línea de trabajo la monografía sobre «Inundaciones en la ciudad y término de Alicante» $\mathrm{o}$ «Avenidas fluviales e inundaciones en la cuenca del Mediterráneo». A partir de este momento, mediados de los años ochenta del pasado siglo, se sucederán las contribuciones científicas sobre eventos extremos del profesor Morales, en las que el papel del ser humano como agente «incentivador» del riesgo será un hilo conductor principal de sus investigaciones, que sabrá transmitir a sus discípulos. Este enfoque será especialmente cultivado en los trabajos sobre las sequías en el sureste peninsular donde se pondrán de manifiesto la relación entre incremento del riesgo y la serie actuaciones territoriales desafortunadas (agrarias, urbano-turísticas) llevadas a cabo en este espacio regional en las últimas décadas.

Un notable volumen de las contribuciones del profesor Morales se ha centrado en el estudio de la actividad agraria, sus manifestaciones paisajísticas, sus dinámicas pasadas y presentes y las nuevas formas de agricultura. Esta línea, que se remonta a su primer trabajo de investigación, referido a la expansión de las áreas regadas desde la huerta tradicional hacia los márgenes del valle del Segura, vuelve a aparecer como parte de su tesis doctoral y, de manera más específica, en el libro La vid y el vino en Jumilla (1976), donde se pone de relieve el interés por el análisis geográfico de la agricultura y sus aprovechamientos, pero también por el estudio de las transformaciones que registran estos paisajes relacionadas con el incremento de caudales para ampliar el regadío y con la introducción de nuevas técnicas de trabajo. Precisamente, será la ampliación, y sobre todo, la creación de nuevos espacios regados en el sureste de España, con la consiguiente introducción de nuevos aprovechamientos y, especialmente, la configuración de una agricultura altamente capitalizada y orientada a la exportación, a la que Morales Gil denomina con la expresión «agricultura de ciclo manipulado», una de las cuestiones que mayor atención ha recibido en su trayectoria. No en vano, ésta última fue la temática que escogió para llevar a cabo, por encargo rectoral, la Lección Magistral de Apertura del curso académico 1997-1998 de la Universidad de Alicante («Aspectos geográficos de la horticultura de ciclo manipulado en España»). Dentro de esta línea de investigación destacan, asimismo, aportaciones en las que analiza los factores, físicos y humanos, que han facilitado la configuración de unos de los espacios de mayor rentabilidad agraria de España y donde se destaca la importancia de la radiación solar, especialmente en invierno, para el desarrollo de la agricultura de vanguardia, en amplias zonas del litoral mediterráneo y del sur peninsular. Asimismo, hace hincapié en la importancia de la accesibilidad a los mercados y en la trascendencia económica y social, en términos de aportación al PIB y creación de empleo, de esta actividad agraria. Se trata, en conjunto, de trabajos que evidencian su visión integral del territorio y su espíritu crítico sobre los hechos que en él se desarrollan, con incorporación de reflexiones concretas sobre los 
estímulos y carencias de estos sistemas agrarios. Un apartado específico, dentro de esta línea de investigación, recibe el tema de las demandas y usos agrarios del agua en España. El incremento de demandas de agua, vinculado a la creación de nuevos regadíos es objeto de análisis desde una óptica en la que el agua es entendida como factor de producción que permite una elevada rentabilidad por unidad de gasto, pero apostando, asimismo, por su uso racional y equilibrado. La eficiencia de los regadíos, la rentabilidad del agua para usos agrarios, tanto desde el punto de vista socio-económico como ambiental, los usos competitivos del agua o las repercusiones sociales y económicas asociadas a la llegada de los caudales del trasvase Tajo-Segura, han sido, por tanto, objeto constante de reflexión a lo largo de su vida académica.

Sin duda, otra de las líneas de trabajo geográfico principales del profesor Morales han sido las investigaciones sobre el papel de las infraestructuras de transporte y los recursos naturales y energéticos en la organización de los espacios regionales. En 1974, publica el estudio sobre «Las comunicaciones en Murcia ante el desarrollo regional» que inaugurará un amplísimo grupo de trabajos dedicados al análisis de las infraestructuras viarias (carreteras, ferrocarril, aeropuertos y puertos) que el profesor Morales considera piezas básicas del desarrollo de los territorios. En estos trabajos no se obvia el papel propositivo de la geografía, en el ánimo de mejorar la situación existente, en relación con la localización de equipamientos y áreas logísticas y el trazado de las redes de comunicación, así como la misma ubicación de infraestructuras energéticas. Las aportaciones que integran esta línea de investigación comprenden tanto artículos específicos como capítulos de libro en monografías regionales o temáticas. En algunos casos se trata de trabajos que derivan de la realización de proyectos y contratos de investigación sobre esta temática (aeropuertos), o son el resultado del encargo de elaboración de ponencias en el marco de reuniones y congresos de Geografía, nacionales o internacionales.

Mención especial merecen los trabajos sobre recursos, usos e infraestructuras del agua, aspecto considerado capital para el funcionamiento de los territorios por el profesor Alfredo Morales. En los trabajos elaborados en esta línea de investigación, a lo largo de su dilatada trayectoria académica, subyacen dos ideas principales: el agua es un recurso natural básico para los territorios y sus sociedades y sólo mediante su uso y gestión prudente se pueden justificar medidas de oferta en aquellos espacios geográficos que presentan posibilidades para el desarrollo de actividades agrarias y turísticas rentables y generadoras de riqueza. Y, por su parte, el agua es un elemento principal en los procesos de ordenación territorial y el planteamiento de nuevos usos en los espacios geográficos debe ser siempre respetuoso con los recursos existentes. Una aportación principal en esta línea de investigación es la publicación coordinada sobre «Demanda y economía del agua en España», donde el profesor Morales hará 
una meditada y ponderada reflexión sobre los trasvases de agua en nuestro país. Dentro de esta línea de trabajo desarrollará, asimismo, varios trabajos sobre el trasvase Tajo-Segura y su significado socioeconómico y territorial para el sureste peninsular. Un apartado especial merece, en este grupo de trabajos sobre temáticas hídricas, las investigaciones sobre sistemas de abastecimiento urbano de agua que tiene en la investigación sobre la Mancomunidad de los Canales del Taibilla, sistema público de abastecimiento de aguas que constituye un hito principal para comprender el desarrollo de un espacio regional tan condicionado por la natural escasez de recursos hídricos y donde se ha hecho necesaria la puesta en marcha, desde fechas tempranas del pasado siglo, de medidas de aportación de caudales y de gestión del recurso.

Otra temática de investigación, considerada básica para la formación de un geógrafo por Alfredo Morales, será la relacionada con la Geografía Física, y en particular con la geomorfología. Destacan en esta línea de trabajo sus primeras aportaciones al conocimiento del medio físico murciano o a los relieves volcánicos canarios, resultado de su estancia como profesor adjunto en la Universidad de La Laguna, y los trabajos sobre geomorfología estructural y climática del territorio alicantino desarrollados tras su llegada al campus alicantino. Estas investigaciones sobre geografía física ocuparán, en esencia, las primeras décadas de su actividad investigadora, pero de modo más o menos directo, a través de la dirección de proyectos de investigación competitivos, será cultivada a lo largo de su trayectoria académica. Un hecho que resultará decisivo para la formación de becarios de investigación y alumnos de especialidad en aspectos geomorfológicos y, en sentido, amplio, geográficos, en el departamento de Análisis Geográfico Regional de la Universidad de Alicante, será la realización de la serie «campañas de otoño» del profesor Dr. Jesús García Fernández, que, iniciadas en 1985, se prolongarán prácticamente hasta los meses previos al propio fallecimiento del maestro castellano. En la organización de estas campañas anuales $\mathrm{y}$, muy especialmente, en la búsqueda de fondos y ayudas de investigación para su realización, tendrá un papel decisivo el profesor Morales. Estas enseñanzas permitirán el desarrollo de una serie de investigaciones sobre geomorfología y biogeografía en el departamento alicantino de Análisis Geográfico Regional, que serán desarrolladas por los profesores del área de geografía física. Debe destacarse, en esta línea de trabajo, las aportaciones llevadas a cabo en la última década en relación con los factores climáticos que han favorecido el desarrollo de una actividad agraria de vanguardia (horticultura de ciclo manipulado) en amplias zonas de nuestro país; pero con especial incidencia en las regiones del sureste ibérico y cuyo significación socioeconómica y territorial debe tanto, en efecto, a la existencia de unas condiciones climáticas muy favorables.

Por último, una línea de investigación, menos conocida, pero de gran querencia por parte del profesor Alfredo Morales ha sido la Geografía Histórica, 
que encuentra aproximaciones de gran interés como los trabajos sobre roturaciones en el Altiplano de Jumilla en el siglo XviII; sobre el municipio de Jumilla en 1755; sobre la propiedad rural en el Altiplano de Jumilla-Yecla en los siglos XVIII y XIX; sobre la creación de los ayuntamientos constitucionales de la Huerta de Murcia en 1820; sobre la desamortización de bienes de rústica en Hellín; sobre la propiedad y tenencia de la tierra en el Campo de Cartagena a comienzos del siglo xx; referidas a iniciativas populares en la construcción de obras hidráulicas de los siglos XVIII y XIX; o centradas en las aportaciones del higienismo de finales del siglo XIX, en la construcción de entornos urbanos más saludables.

J. Fernando Vera Rebollo Jorge Olcina Cantos

María Hernández Hernández Alicante, enero de 2016 